\title{
PARADOJAS DE LA COSTUMBRE JURÍDICA *
}

\author{
Damiano Canale
}

Universidad de Bocconi

RESUMEN. En este artículo, el autor, inspirándose en la interpretación propuesta por KRIPKE de algunos pasajes de las Investigaciones Filosóficas de Wittgenstein, discute algunas paradojas en las que, en su opinión, incurren el discurso de la ciencia jurídica y el de la doctrina cuando identifican al usus y a la opinio iuris seu necessitatis como elementos esenciales de la costumbre jurídica.

Palabras clave: costumbre, regla social, convención, usus, opinio iuris, opinio necessitatis.

ABSTRACT. Under the light of KRIPKE'S interpretation of some paragraphs of Wittgenstein's Philosophical Investigations, the author of this paper denounces the paradoxes resulting, in his opinion, when usus and opinio iuris seu necessistatis are considered - as legal science and doctrine donecessary elements in the identification of legal customs.

Keywords: customs, social rule, convention, usus, opinio iuris, opinio necessitatis.

* Fecha de recepción: 31 de julio de 2009. Fecha de aceptación: 3 de septiembre de 2009.

Este ensayo constituye la versión revisada y ampliada de la relación presentada en el congreso: «La consuetudine giuridica: storia, teoria, ambiti disciplinari», llevado a cabo el 20 de septiembre de 2007 en la Università Statale di Milano. Deseo agradecer a G. ZACCARIA y R. GuASTINI sus observaciones. También me siento agradecido con G. TUZET y E. FITTIPALDI por la discusión de algunas de las tesis aquí sostenidas. 
«Things are made to look the same only when we fail to examine them too closely».

(N. CARTWRight)

\section{INTRODUCCIÓN}

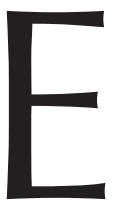

n este ensayo me propongo discutir algunas paradojas en las que incurre el discurso de la ciencia jurídica y la doctrina sobre la costumbre jurídica. Se trata de paradojas bien conocidas por el filósofo; su origen y sus implicaciones no representan sorpresas para él. De éstas, la ciencia jurídica y la doctrina, parece, sin embargo, que no son conscientes, con el resultado de que en su discurso la costumbre jurídica se vuelve frecuentemente un término vacío, útil solamente, como se ha sostenido, para abrir «un cierto espacio de discreción» a favor de los operadores jurídicos ${ }^{1}$.

Las paradojas que ilustraré aquí a continuación se inspiran en la interpretación propuesta por S. KRIPKE de algunos pasajes centrales de las Investigaciones filosóficas de L. WitTGENSTEIN ${ }^{2}$. En este sentido, podemos decir que se trata de paradojas al estilo «kripkensteiniano». Su estudio permite evidenciar cómo el término «costumbre jurídica» es, sin lugar a dudas, vago y ambiguo, y cómo dicha vaguedad y ambigüedad generan contradicciones, dilemas, calambres mentales, que hacen deseable un atento trabajo de redefinición por parte de la ciencia jurídica ${ }^{3}$.

\section{LOS REQUISITOS DE LA COSTUMBRE ENTENDIDA COMO TIPO DE PRODUCCIÓN DE NORMAS JURÍDICAS}

El discurso de la ciencia jurídica y de la doctrina es particularmente relevante para comprender en qué consiste una costumbre jurídica. Esto se debe en primer lugar al hecho de que los ordenamientos no proveen generalmente una definición de «costumbre jurídica», incluso en el caso en que pertenezca al ordenamiento una norma sobre la producción de normas que califique a la costumbre como fuente del Derecho; tal norma habitualmente no establece en qué condiciones una regularidad de conducta, como quiera que esté calificada, constituye un caso de producción de normas jurídicas.

1 B. Celano, «Consuetudine e norme sulla produzione di norme», en P. Comanducci y R. GUASTINI (eds.), Struttura e dinamica dei sistemi giuridici, Torino, Giappichelli, 1996, trad. castellana de J. J. MorESO, «Costumbres y normas sobre la producción de normas», en B. CELANO, Dos estudios sobre la costumbre, México, Fontamara, 2000, p. 115.

2 Cfr. S. KRIPKE, Wittgenstein on Rules and Private Language. An Elementary Exposition, Oxford, Blackwell, 1982. Por «paradoja» entiendo un argumento en el que las premisas son consideradas verdaderas, la regla de inferencia es considerada válida, pero la conclusión aparece como falsa.

3 Por «redefinición» entiendo la precisión del contenido de un término que, «aunque permanezca en el ámbito de los [sus] significados usuales», trata de reducir «la ambigüedad y la indeterminación atribuyendo a la palabra aquel significado... que consienta la más correcta formulación de los problemas que requieren el empleo de la palabra definida» (A. BELVEDERE, «Aspetti ideologici delle definizioni nel linguaggio del legislatore e dei giuristi», en VV.AA., Definizioni giuridiche e ideologie, Milano, Giuffrè, 1979, p. 355). 
De tal modo, como ha subrayado V. CRISAFULLI, estamos ante un tipo de reenvío, por parte de los ordenamientos, a la reflexión de los juristas, de modo que se hace difícil distinguir «la figura dogmática de la costumbre, fuente formal del ordenamiento determinado», de la «respectiva noción "doctrinal" predominante en el ámbito de la teoría general» ${ }^{4}$. Pero ¿cuál es esta noción doctrinal predominante en los sistemas de civil law? ${ }^{5}$.

Las condiciones para que una norma consuetudinaria exista son tradicionalmente dos: el usus, dicho también diuturnitas o longa repetitio ${ }^{6}$, y la opinio iuris seu necessitatis. La primera condición es generalmente definida como «elemento objetivo» o «material» de la costumbre jurídica, la segunda como «elemento subjetivo»o «psicológico». Detengámonos a considerar estas condiciones con mayor atención.

\section{USUS}

El elemento objetivo o material de la costumbre se articula tradicionalmente con las siguientes condiciones, es decir, la repetición de una conducta genera una norma consuetudinaria $\mathrm{si}^{7}$ :

a) La conducta se lleva a cabo por la generalidad de los individuos que se encuentran en la situación regulada (generalidad).

b) La conducta se lleva a cabo sin excepciones (uniformidad).

c) La conducta se lleva a cabo sin interrupciones (continuidad).

d) La conducta se repite con pequeños intervalos entre una y otra (frecuencia).

e) La conducta no se lleva cabo secretamente (publicidad).

No analizaré lo relativo a la vaguedad de los requisitos del usus; por tanto, no me ocuparé de las modalidades, con frecuencia problemáticas, a través de las cuales se verifica si tales requisitos son satisfechos. El problema de la verificación de la costumbre es ciertamente relevante y delicado. Se trata, sin embargo, de un problema que no tiene inmediata relevancia conceptual. Las condiciones de tal verificación, en efecto, mutan en función del concepto de costumbre jurídica adoptado. Me parecen muy interesantes para tal propósito dos paradojas que el requisito del usus desencadena en el contexto que se examina. Estas paradojas —que llamaré, respectivamente, de la

4 V. CRISAFUlLI, Lezioni di diritto costituzionale. II: L'ordinamento costituzionale italiano. I: Le fonti normative, Padova, CEDAM, 1993, p. 161.

5 En este ensayo me ocuparé de los usos del término «costumbre jurídica» en la ciencia y en la doctrina europeo-continentales, en particular en la italiana y en la alemana. No me ocuparé, entonces, de los requisitos de la costumbre jurídica en el ámbito de los sistemas de common law, puesto que el tema requeriría un tratamiento independiente. Vid., sobre este punto, A. CROMARTIE, «The Idea of Common Law as Custom», en A. Perreau-Saussine y J. B. Murphy (eds.), The Nature of Customary Law. Legal, Historical and Philosophical Perspectives, Cambridge, Cambridge University Press, 2007, pp. 203 y ss.; R. SHINER, Legal Institutions and the Sources of Law, Berlin-New York, Springer, 2005; A. WATSON, «An Approach to Customary Law», University of Illinois Law Review, vol. 3, 1996, pp. 561-576.

${ }^{6}$ Por razones de simplicidad trataré estos términos como sinónimos, si bien éstos denotan requisitos diversos de las conductas reguladas por la costumbre jurídica.

${ }^{7}$ Cfr. H. O. FreITAG, Gewohnheitsrecht und Rechtssystem, Berlin, Duncker \& Humbolt, 1975, pp. 15 y ss.; N. BobBio, voz «Consuetudine (teoria generale)», Enciclopedia del diritto, vol. IX, Milano, Giuffrè, 1961, p. 428 . 
derrotabilidad temporal y de la derrotabilidad material — constituyen el núcleo de este ensayo. Las consideraciones que se siguen de ellas se limitan a especificar su contenido, proyectar las hipótesis de solución y profundizar sus implicaciones para otros aspectos de la costumbre jurídica.

\subsection{Paradoja de la derrotabilidad temporal}

Supongamos que los proveedores de los servicios de Internet garanticen espontáneamente, desde el momento en que tales servicios se hayan introducido en el mercado, la privacidad de los registros de conexión del cliente. Supongamos que tal conducta se lleve a cabo de manera general, uniforme, continua, frecuente y pública. Supongamos, entonces, que tal conducta genere una norma consuetudinaria praeter o secundum legem — según el ordenamiento jurídico que se considere- que se presta a ser así formulada.

(1) El proveedor de servicios de Internet tiene la obligación de garantizar la privacidad de los registros de conexión del cliente.

No obstante, si observamos bien, desde el momento en que tiene lugar la conducta llevada a cabo por los proveedores de servicios de Internet hasta el momento actual $t_{1}$ resulta lógicamente deducible también una norma consuetudinaria diversa.

(2) El proveedor de servicios de Internet tiene la obligación de garantizar la privacidad de los registros de conexión del cliente hasta $t_{1}$, pero no tiene la obligación de garantizar la privacidad de tales registros sucesivamente a $t_{1}$.

Aunque pueda parecer sorprendente, la conducta llevada a cabo en el momento actual por parte de los proveedores es conforme tanto a la norma (1) como a la norma (2). Atendiendo a (1), sin embargo, subsiste para los proveedores la obligación de la privacidad también sucesivamente a $t_{1}$; atendiendo a (2), en cambio, tal obligación no subsiste sucesivamente a $t_{1}$, tal vez a la luz de una mutación imprevista en el contexto de acción (pensamos, por ejemplo, en un atentado terrorista, realizado con la ayuda del Internet, que justifique que tal obligación pierda importancia).

Conviene entonces preguntarse: ¿qué norma consuetudinaria se produce del simple comportamiento repetitivo de los proveedores? ¿la norma (1) o la norma (2)? La respuesta está indeterminada.

Podríamos decir que el desafío lanzado por la paradoja de la derrotabilidad temporal es la siguiente: es siempre posible poner en duda que una conducta actual sea conforme a una cierta regla, aunque tal conducta nos parezca una simple repetición de las conductas que en el pasado constituían casos de observación de aquella regla.

Esta tesis puede ser formulada también de una manera diferente: ningún hecho de mi vida pasada asegura que yo esté siguiendo una cierta regla ${ }^{8}$.

${ }^{8}$ Cfr. KRIPKE, Wittgenstein on Rules and Private Language, cit., p. 55. 


\subsection{Paradoja de la derrotabilidad material (o tesis de la textura abierta de las prácticas sociales)}

Retomemos el ejemplo apenas propuesto y supongamos que la repetición general, uniforme, continua, frecuente y pública del comportamiento de los proveedores de servicios de Internet genere, prima facie, la norma consuetudinaria (1). Supongamos ahora que se tome en consideración una propiedad de la conducta regulada que hasta este momento no habíamos considerado. Por ejemplo, el hecho de que el cliente en cuestión haya sido condenado con una sentencia firme por tráfico de imágenes de pornografía infantil a través de Internet. A la luz de la relevancia atribuida a esta propiedad, la repetición del comportamiento de los proveedores es idónea para generar dos normas consuetudinarias que imputan al mismo caso consecuencias jurídicas diversas:

1. El proveedor de servicios de Internet, también en el caso en que el cliente haya sido condenado por tráfico de imágenes de pornografía infantil a través de Internet, tiene la obligación de garantizar la privacidad del registro de las conexiones del cliente.

2. El proveedor de servicios de Internet, en el caso en que el cliente haya sido condenado por tráfico de imágenes de pornografía infantil a través de Internet, no tiene la obligación de garantizar la privacidad del registro de las conexiones del cliente.

La elección de la norma consuetudinaria generada por la conducta de los agentes será determinada por la relevancia atribuida a la propiedad hasta aquel momento no considerada.

La atribución de relevancia a tal propiedad tiene, en efecto, fuerza retroactiva; ésta se remonta hacia atrás en la serie de conductas que se repiten, determinando qué conductas pertenecen desde el inicio a aquella serie, y qué conductas, en cambio, nunca han formado parte de ella9 .

También en el caso recién considerado, por tanto, la repetición calificada de una misma conducta se presta a generar normas consuetudinarias de contenido diverso. Así como se ha observado en relación con la paradoja de la derrotabilidad temporal, tal conclusión se sigue del hecho de que es siempre posible poner en duda que la realización de una cierta conducta constituya un caso de observancia de una cierta regla. La paradoja de la derrotabilidad material, sin embargo, enriquece esta consideración con un elemento ulterior. Ésta muestra cómo se puede dudar de la relación que existe entre una regla y una conducta también en el caso de que tal conducta sea susceptible de ser descrita prima facie como conforme a aquella regla. Las propiedades de la conducta que constituye un caso de observancia de la norma consuetudinaria, en efecto, no son individuadas por la norma misma sino que más bien contribuyen a individuarla.

Esta tesis se presta a ser formulada también en modo diverso: ninguna regla que haya yo seguido en el pasado asegura que una cierta conducta consista en seguir aquella regla.

9 La fuerza retroactiva de la atribución de relevancia caracteriza, como es sabido, la derrotabilidad conceptual descrita por H. HART, «The Ascription of Responsibility and Rights», Proceedings of the Aristotelian Society, vol. 49, 1949, pp. 171-194. 


\subsection{Conductas ejemplares}

Consideremos ahora un tercer problema que afecta al requisito del usus, cuya discusión podría quizá sugerir la solución de las dos paradojas apenas consideradas.

En algunas ramas del Derecho, como por ejemplo en el Derecho internacional, o en el Derecho constitucional, la doctrina admite la llamada «costumbre instantánea», o sea, una norma consuetudinaria que surge en correspondencia con un comportamiento concreto ${ }^{10}$.

Considerar una conducta concreta como un supuesto de hecho idóneo para producir una norma consuetudinaria parece confutar la hipótesis según la cual el usus constituye una condición necesaria, aunque no suficiente, de la producción de dicha norma ${ }^{11}$.

En mi opinión, en este caso el término «repetición» asume simplemente una connotación diversa y más interesante desde el punto de vista filosófico. Se trata, en efecto, de una repetición orientada al futuro y no al pasado, es decir, de una «expectativa de repetición» generada en el grupo de una conducta concreta. Tratemos de aclarar este punto.

Según el clásico planteamiento humano, implícitamente aceptado por la ciencia jurídica y la doctrina hasta nuestros días, la repetición no constituye una propiedad del comportamiento que se repite sino el resultado de una modificación perceptiva por un observador externo; es decir, la repetición no sería una propiedad de ciertos hechos o estados de cosas, sino una apreciación del sujeto que los observa ${ }^{12}$.

Es posible, sin embargo, concebir la repetición en modo diverso, o sea, como una propiedad social de un cierto comportamiento, que consiste en la capacidad de aquel comportamiento de servir de «ejemplo». Como muestra nuestra experiencia cotidiana, en efecto, lo que caracteriza a un ejemplo es que éste vale para todos los casos del mismo tipo y, al mismo tiempo, está incluido entre ellos. El ejemplo es un hecho, un estado de cosas o un evento que es tratado como «modelo» de cada hecho, estado de cosas o evento del mismo tipo. En el caso de la costumbre, el comportamiento ejemplar explicita, si es posible decirlo así, una regla social implícita, pero no formula lingüísticamente tal regla, más bien representa mediante un comportamiento concreto la conducta que aquella prescribe.

${ }^{10}$ En la doctrina internacionalista, la noción de costumbre instantánea se basa «on the view that an opinio juris expressed by the entire community of States will itself validate a rule of law» (C. F. AMERASINGHE, Principles of Institutional Law of International Organizations, Cambridge, Cambridge University Press, 2005², p. 190). Vid., sobre este punto, H. T. KLAMI, «Gewohnheitsrecht als Methodenproblem», en N. ACHTERBERG (hrsg.), Rechtssprechungslebre, Köln, Heymann, 1986, p. 360; G. ARANGIO RUIZ, voz «Consuetudine. III) Consuetudine internazionale», Enciclopedia giuridica, vol. VIII, Roma, Istituto della Enciclopedia Italiana, 1988, pp. 7 y 10. Según Arangio Ruiz, en particular, la opinio es idónea para compensar la falta del usus y el usus, a su vez, es idónea para compensar la falta de la opinio.

${ }^{11}$ Así, por ejemplo, P. ZICCARDI, voz «Consuetudine (diritto internazionale)», Enciclopedia del diritto, vol. IX, Milano, Giuffrè, 1961, p. 493.

${ }_{12}$ Según Hume la repetición, a la par de la necesidad de algo para seguir otra cosa, «is something that exists in the mind, not in objects; nor is it possible for us ever to form the most distant idea of it, consider'd as a quality of bodies» [D. Hume, A Treatise of Human Nature (1739-1740), Oxford, Clarendon Press, 1967, pp. 163-164]. Esta tesis está bien expresada, en relación con la costumbre jurídica, en C. Rossano, La consuetudine nel diritto costituzionale. I: Premesse generali, Napoli, Jovene, 1992, pp. 71-72. 
Al traducir esta observación al léxico de la filosofía de la mente, podemos decir que la conducta ejemplar es aquella conducta que tiene la propiedad de generar, en un cierto campo de acción, por un lado la creencia de que en el pasado se habría debido actuar del mismo modo en casos similares, aunque esto no haya ocurrido, y por el otro, el deseo de actuar así en el futuro. Es decir, que cabe describir la repetición en términos intencionales, tratándola como una propiedad motivacional de una conducta; una conducta está dotada de esta propiedad si se trata de una instancia en el mundo de las razones para actuar que son el fundamento de fenómenos sociales tales como la imitación, la costumbre, etcétera ${ }^{13}$.

Por lo menos con referencia a las costumbres de Derecho público y a la costumbre internacional, esto me parece un modo filosóficamente más interesante de caracterizar el requisito del usus.

De todos modos, lo que acabamos de observar deja abierto el problema insistentemente señalado en literatura, de la distinción entre reglas sociales y normas jurídicas, un problema que el criterio del usus no permite resolver ${ }^{14}$. ¿La repetición calificada de «cualquier» conducta social genera una norma consuetudinaria? La respuesta es obviamente negativa. No todas las reglas o regularidades sociales son consideradas idóneas para producir normas jurídicas, ni dicha idoneidad constituye una condición suficiente para decretar el nacimiento de tales normas.

Para resolver este problema, ciencia jurídica y doctrina individuan tradicionalmente una segunda condición necesaria para el nacimiento de una norma consuetudinaria: la opinio iuris seu necessitatis.

\section{OPINIO IURIS SEU NECESSITATIS}

A diferencia del usus, el requisito de la opinio no es simplemente vago sino también ambiguo, es decir, no es solamente impreciso sino que asume sentidos diversos en el discurso de la dogmática y de la doctrina. Me ocuparé sólo de algunos de ellos, partiendo para tal fin de una clasificación propuesta por N. BOBBIO aún hoy ampliamente utilizada en la literatura ${ }^{15}$.

\subsection{Opinio iuris como creencia del grupo en la obligatoriedad jurídica de la conducta}

Es ésta la acepción más común de la opinio en el debate contemporáneo. De ella se dan al menos dos versiones, que caracterizaré, con ánimo de simplificar, utilizando la distinción lexical entre «opinio iuris» $\mathrm{y}$ «opinio necessitatis».

13 Así debe subrayarse que el problema de fondo que afecta a cualquier explicación de estos fenómenos consiste en el hecho «that explanations in terms of habit are, in a sense, "self-fulfilling". When we refer to a babit, we refer to an entity that includes the very action that is being explained» (B. POLLARD, «Actions, Habits and Constitution», Ratio, vol. 19, 2006, p. 239).

14 N. BobBio, La consuetudine come fatto normativo, Padova, CEDAM, 1941, p. 69.

15 Cfr. BobBio, voz «Consuetudine», cit., pp. 429 y ss. 
Con el término «opinio iuris», la ciencia jurídica y la doctrina se refieren, preferentemente, a la creencia en la obligatoriedad de la norma consuetudinaria, cuya observancia se instancia en la repetición calificada de una conducta. El término «opinio necessitatis», en cambio, hace siempre referencia a la creencia en la obligatoriedad de una regla social, cuya observancia se presenta en la repetición calificada de una conducta que tiene contenido idéntico al de la norma consuetudinaria. Consideremos más de cerca estos dos requisitos.

\subsubsection{Paradoja de la falsa creencia}

Si partimos de la primera acepción del requisito de la opinio, una regularidad de conducta general, uniforme, continua, frecuente y pública, genera la norma consuetudinaria $\mathrm{N}$ si, y sólo si, los agentes creen que tal conducta es obligatoria, conforme a $\mathrm{N}$.

Formulada en estos términos, sin embargo, la definición considerada es circular porque la conclusión está ya contenida en las premisas ${ }^{16}$. Tal vicio argumentativo viene generalmente superado en la literatura mediante la llamada «teoría del error»: los agentes creen que la conducta es jurídicamente vinculante, pero se trata de una creencia falsa, ya que la norma consuetudinaria $\mathrm{N}$ aún no existe. Sin embargo, este error motiva el comportamiento general, uniforme, continuo, frecuente y público que genera $\mathrm{N}^{17}$.

Ahora bien, recurrir a la teoría del error para aclarar el concepto de costumbre jurídica conduce a un resultado paradójico. Según esta tesis, en efecto, los actores sociales creen falsamente que existe una norma jurídicamente vinculante y, en consecuencia, se comportan conforme a ella. Pero tal norma existe si, y sólo si, el presupuesto de la opinio iuris es satisfecho, o sea, si la creencia de los actores sociales es verdadera. De esto se deriva la conclusión de que la falsedad de una creencia es una de las condiciones que la hacen verdadera.

Se podría fácilmente replicar que lo recién expuesto es una paradoja (lógica) sólo aparentemente. La teoría del error permite, en efecto, explicar en modo coherente la genesi de las normas consuetudinarias. Asumamos que, prima facie, un proveedor de servicios de Internet crea jurídicamente obligatorio garantizar la privacidad del registro de las conexiones del cliente y se comporte en consecuencia, aunque tal creencia sea falsa. Asumamos que otros proveedores, apoyándose en el comportamiento del primer proveedor, desarrollan la misma (falsa) creencia y se comportan del mismo modo. La conducta general, uniforme, continua, frecuente y pública que le seguirá decreta el nacimiento de una norma consuetudinaria que prescribe tal conducta. Si se entiende en estos términos, parece que la teoría del error no llega a conclusiones paradójicas y permite más bien mostrar cómo a partir de una regularidad social puede surgir una costumbre jurídica.

Los defensores de la teoría del error, sin embargo, confunden una explicación genética, como la apenas ilustrada, con una explicación analítica, atribuyendo impropiamente a la primera la función que corresponde a la segunda.

16 Cfr. H. KeLSEn, Teoria generale del diritto e dello Stato, Milano, ETAS, 1994, p. 115.

17 Cfr. F. Modugno, voz «Fonti del diritto (diritto costituzionale)», Enciclopedia giuridica, vol. XIV, Roma, Istituto della Enciclopedia Italiana, 1988, p. 12. 
Una explicación genética explica el concepto $\mathrm{Y}^{18}$ a partir de la construcción de un bicondicional que tiene grosso modo la forma siguiente: «X tiene la propiedad $\mathrm{Y}$ si y sólo si es generalmente considerado $\mathrm{Y}$ en las circunstancias normales $\mathrm{Z} \gg{ }^{19}$. Ahora bien, este bicondicional no provee un análisis de $\mathrm{Y}$, porque $\mathrm{Y}$ está presente tanto en el lado izquierdo como en el lado derecho del bicondicional. Dicha tesis es por lo tanto circular. No se trata, sin embargo, de una circularidad viciosa, o por lo menos no lo es necesariamente. Esta explicación se presta, en efecto, a acrecentar nuestro conocimiento de $\mathrm{Y}$ explicando como llegamos a poseer el concepto $\mathrm{Y}$ o bien la cadena causal que produce el hecho, el estado de cosas o el evento a que dicho concepto se aplica. Es ésta la prestación cognoscitiva proporcionada por la teoría del error, la cual ilustra, en modo aparentemente persuasivo, cómo nace una costumbre jurídica.

Una explicación analítica, en cambio, explica el concepto Y construyendo un bicondicional del tipo «X tiene la propiedad Y si y sólo si tiene la propiedad Z». Este bicondicional no es circular y explicita en qué consiste poseer Y, o sea la articulación o la naturaleza de un concepto.

Ahora bien, no está aquí en discusión el carácter informativo de las explicaciones genéticas ni su evidente utilidad para las ciencias sociales. La objeción que quisiera dirigir a la teoría del error es otra. En primer lugar, esta teoría no explica ni la articulación ni menos aún la naturaleza del concepto «costumbre jurídica», sino que, en un cierto sentido, la presupone. En segundo lugar, en caso de que sea impropiamente atribuida a esta teoría tal función explicativa, aquélla asume la forma contradictoria «X tiene la propiedad Y si y sólo no tiene la propiedad Y». Pero si una explicación genética es analíticamente contradictoria, entonces pierde toda capacidad explicativa. Ésta no sólo no nos dice algo en relación con la articulación o la naturaleza de un concepto, sino que además termina por no explicar su génesis. Una explicación acerca del modo en que llegamos a poseer un concepto carece en efecto de sentido si no queda claro en qué consiste poseerlo ${ }^{20}$.

La paradoja lógica que estamos considerando puede ser resuelta adoptando dos estrategias alternativas que imponen afinar o bien revisar la teoría del error.

18 Asumo aquí, para simplificar, que los conceptos expresan propiedades, sin tomar sin embargo posición sobre qué tipo de propiedades expresen los conceptos.

${ }^{19} \mathrm{La}$ distinción entre explicaciones genéticas y explicaciones analíticas es generalmente utilizada en la literatura (sobre la base de la celebre crítica, formulada por parte QuINE, a la distinción analítico/sintética) para evidenciar la aportación cognitiva proporcionada por las primeras y las conclusiones reduccionistas a las cuales se agregarían las segundas. Cfr., por ejemplo, P. PETTiT, «Affirming the Reality of Rule-Following», Mind, vol. 99, 1990, pp. 433-439; H. PRICE, Facts and the Function of Truth, Oxford, Blackwell, 1988, passim; S. BlackBuRn, Spreading the Word, Oxford, Oxford University Press, 1984, pp. 210 y ss. Tal distinción se retoma aquí con un objetivo muy diverso. Sobre la base de lo que he sostenido es posible afirmar que también las explicaciones analíticas se prestan a ampliar el conocimiento; éstas simplemente dan acceso a un tipo de conocimiento diferente de aquel al que conduce una explicación genética. En el estudio de los conceptos jurídicos, por ejemplo, esta afirmación está justificada en el caso en que el contenido conceptual sea concebido no en términos causales sino en términos inferenciales, $c f r$. D. CANALE, «Inferenzialismo semantico e ragionamento giuridico», Ragion pratica, núm. 25, 2005, pp. 301-334.

${ }^{20}$ De tal consideración se sigue que no es posible dar una explicación de la costumbre jurídica en términos estrictamente causales, como la teoría del error parece presumir. Si se le pide especificar a cuáles condiciones una cierta conducta es conforme a una costumbre jurídica, en efecto, estamos obligados a especificar las características de la misma conducta. La norma consuetudinaria y la conducta conforme a ésta no gozan de existencias separadas desde el punto de vista lógico y, por tanto, una no puede ser causa de la otra. 


\subsubsection{Solución de la diferencia temporal}

Supongamos que en $\mathrm{t}_{1}$ la generalidad de los agentes tenga la creencia $\mathrm{C}$ de que existe la norma consuetudinaria $\mathrm{N}$, aunque $\mathrm{N}$ aún no exista en $t_{1} \mathrm{y} \mathrm{C}$ sea por tanto falsa. Supongamos que, sin embargo, $\mathrm{C}$ motive a los agentes una conducta general, uniforme, constante y pública que se repite en $t_{2}, t_{3} \ldots t_{n}$. La repetición de esta conducta hace, por tanto, a $\mathrm{C}$ verdadera en $t_{n}$, generando la norma consuetudinaria $\mathrm{N}$. Reconstruido en estos términos, el argumento de la falsa creencia no conduce a una conclusión contradictoria: no se da el caso de que la creencia de obligatoriedad sea verdadera y falsa al mismo tiempo y bajo el mismo aspecto. En el ejemplo propuesto, en efecto, $\mathrm{C}$ es falsa en $t_{1}$ y verdadera en $t_{n}$. Los estados de cosas que en $t_{1}$ y $t_{n}$ hacen respectivamente falsa y verdadera $C$, además, no son equivalentes; en $t_{n}$ la conducta creída como obligatoria sí se repite en modo general, uniforme, continuo, frecuente y público, mientras en $t_{1}$ esto no ha ocurrido aún.

Perfeccionada de este modo, la teoría del error escapa, cierto, a la paradoja de la falsa creencia; sin embargo, pierde, al mismo tiempo, buena parte de su propia capacidad explicativa.

Para comprender este punto, es oportuno retomar las consideraciones desarrolladas en relación con el requisito del usus. En aquella ocasión habíamos sostenido que es siempre posible dudar de que una conducta sea conforme a una norma consuetudinaria N. Esto porque el hecho de que $\mathrm{N}$ exista o no puede ser comprobado solamente a posteriori, después de que la conducta regulada por $\mathrm{N}$ se haya repetido en modo calificado. Ahora bien, si la existencia de $\mathrm{N}$ puede ser comprobada solamente $a$ posteriori, con mayor razón podrá ser comprobado solamente a posteriori si la creencia de $\mathrm{C}$ en la existencia de $\mathrm{N}$ es verdadera o falsa. ¿A qué conclusión conduce entonces este argumento? Es posible dudar de que la creencia $\mathrm{C}$ esté en la base de la norma consuetudinaria $\mathrm{N}$, porque $\mathrm{C}$ se presta a motivar una conducta que constituye un caso de aplicación de una norma consuetudinaria de contenido diverso.

Retomemos el ejemplo propuesto precedentemente y supongamos que un proveedor de servicios de Internet crea en $t_{1}$ que subsiste la obligación jurídica de garantizar la privacidad del registro de los clientes, aunque tal creencia sea falsa, desde el momento en que en $t_{1}$ no existe norma consuetudinaria alguna que prescriba tal conducta. Supongamos que, sin embargo, tal creencia motive al proveedor a garantizar la privacidad del registro de los clientes, y que su conducta genere en los otros proveedores la misma (falsa) creencia, motivando la repetición con los requisitos debidos de la misma conducta. Como habíamos observado en los párrafos precedentes, la repetición general, uniforme, continua, frecuente y pública de tal conducta se presta a generar, en $t_{n}$, tanto la norma (1), con base en la cual los proveedores de servicios de Internet tienen la obligación de garantizar la privacidad de las conexiones del cliente, como la norma (2), con base en la cual los proveedores de servicios de Internet tienen la obligación de garantizar la privacidad de las conexiones del cliente hasta $t_{n}$, mientras tal obligación no subsiste después de $t_{n}$. En el caso de que la repetición de la conducta genere la norma (2) en lugar de la norma (1), por tanto, la creencia C con base en la cual tal obligación subsistiría, se revelará falsa tanto en $t_{1}$ como en $t_{n}$. 
¿Qué implicaciones tiene este argumento para la discusión de la teoría del error? No parece constituir una condición necesaria de la costumbre jurídica el hecho que la creencia errónea de los agentes en $t_{1}$ motive la repetición debidamente cualificada de una conducta que haga verdadera esta misma creencia en $t_{n}$. Se da, en efecto, el caso de normas consuetudinarias que nacen de la errónea creencia de los agentes pero el contenido es diferente de aquél que tienen las normas consideradas obligatorias.

Si lo que hasta aquí hemos observado es correcto, entonces, cualquier creencia que sea capaz de motivar la repetición debidamente cualificada de una conducta satisface, paradójicamente, el requisito de la opinio iuris. Se deriva que la teoría del error, por un lado, se reduce a una mera conjetura psicológica formulada a posteriori, y por el otro, resulta irrelevante para identificar la norma consuetudinaria, desde el momento en que cualquier creencia parece ser capaz de generar una.

\subsubsection{Solución de la diferencia de contenido}

Asumamos que en $t_{1}$ la generalidad de los agentes tengan la creencia $\mathrm{C}$ de que la regla social $n$ sea obligatoria, incluso si $n$ no es una norma consuetudinaria. $C$ es por tanto falsa si tiene por objeto la existencia de una norma consuetudinaria $\mathrm{N}$, pero es verdadera si tiene por objeto la existencia de una regla social $n$. Asumamos, efectivamente, que $\mathrm{C}$ motive en los agentes una conducta general, uniforme, continua, frecuente y pública que suponga la observancia de $n$ en $t_{2}, t_{3} \ldots t_{n}$. La repetición de esta conducta, motivada por la creencia en su obligatoriedad, generará entonces la norma consuetudinaria $\mathrm{N}$ que tiene contenido idéntico a $n$.

La paradoja de la falsa creencia atribuye a la creencia de la obligatoriedad un contenido diverso de la existencia de una norma consuetudinaria. Esta estrategia teórica se resuelve, sin embargo, en una declinación diversa de la opinio, entendida no como opinio iuris sino como una opinio necessitatis.

\subsection{Opinio necessitatis y enforcement de una regla social}

Como se acaba de indicar, el requisito de la opinio necessitatis se diferencia del requisito de la opinio iuris porque la creencia en la obligatoriedad, en este caso, no tiene por objeto una norma jurídica sino una regla social relativa a un interés merecedor de tutela. Reformulada a la luz de esta acepción de la opinio, la expresión «costumbre jurídica» se refiere, por tanto, al siguiente estado de cosas: una conducta se repite en modo general, uniforme, frecuente y público en el interior de un cierto contexto social y es acompañada por un comportamiento de los agentes que consiste en asumir aquella conducta como un estándar vinculante. Se trata de un supuesto de hecho que, bien visto, presenta las mismas características distintivas de las social rules descritas por $\mathrm{H}$. HART ${ }^{21}$. Según HART, en efecto, una regla social existe a condición de que un com-

${ }^{21}$ Como ha señalado oportunamente S. PERRY, «Hart's analysis of a social rule is meant to make more precise the familiar idea of a rule or norm which is based on custom» (S. PERRY, «Hart on Social Rules and the Foundation of the Law: Liberating the Internal Point of View», Fordham Law Review, vol. 75, núm. 3, 2006, 
portamiento sea generalmente llevado a cabo por los miembros de un grupo social, las desviaciones den lugar a la crítica, que tal crítica sea justificada y que, al final, dicho comportamiento se asocie a una «reflective critical attitude» que entienda tal comportamiento como un estándar común de conducta al cual cada uno tiene buenas razones para conformarse ${ }^{22}$.

El requisito de la opinio necessitatis conduce entonces a concebir a la costumbre jurídica como enforcement jurídico de una regla social. El concepto de costumbre jurídica, considerado a la luz de tal requisito califica una regla social, bajo ciertas condiciones, como caso de producción de una norma jurídica dotada del mismo contenido prescriptivo.

El problema, una vez más, consiste en precisar cuáles son tales condiciones. Si la definición de «opinio necessitatis», como se ha señalado, se limita a explicitar en qué consiste una regla social, es necesario aclarar en qué circunstancias el enforcement jurídico de dicha regla está justificado.

\subsubsection{Opinio como voluntas populi}

La solución tradicional de este problema viene provista por la noción de opinio como voluntas populi. Se trata de una acepción del término «opinio» que se remonta a la doctrina romano-canónica de la costumbre como tacitus consensus populi, que encuentra renovada atención a partir del Gewohnheitsrecht de PUCHTA para adquirir después una connotación psicológica en la obra de JHERING y JELLINEK ${ }^{23}$. Esta misma acepción sobrevive hoy en la doctrina canónica y en las teorías de la costumbre jurídica como fuente extra ordinem ${ }^{24}$.

La formalización del requisito de la voluntas populi es tradicionalmente la siguiente: una regla social produce una norma jurídica de contenido idéntico si el pueblo o, más genéricamente, la colectividad quiere que esto suceda ${ }^{25}$.

Cabe subrayar cómo esta solución al problema del enforcement jurídico de las reglas sociales si bien es cierto que amerita atención por parte del historiador del Derecho, no precisa de tanta por parte de la ciencia jurídica y de la doctrina. El requisito de la opinio como voluntas populi resulta en efecto muy problemático. Es suficiente mencionar aquí dos órdenes de problemas conectados entre sí. En primer lugar, el requisito de la voluntas populi admite algunas entidades ontológicas muy controvertidas,

p. 1178). Subrayan la afinidad del análisis hartiano de las reglas sociales con la noción de costumbre jurídica también B. CELANO, Costumbres y normas sobre la producción de normas, cit., p. 98; O. ORESTANO, «Dietro la consuetudine giuridica», en id., «Diritto». Incontri e scontri, Bologna, Il Mulino, 1981, pp. 423-438.

22 H. L. A. HART, Il concetto di diritto, Torino, Einaudi, 1991, pp. 55-57.

${ }^{23}$ Cfr. D. 1,3,32,1; G. F. PuchTA, Das Gewohnheitsrecht, vol. I, Erlangen, Palm, 1828, pp. 53 y ss., 99, 152 y ss.; R. vON IHERING, Das Trinkgeld, Braunschweig, Wetermann, 1882, p. 10; G. JellineK, Allgemeine Staatslebre, Bad Homburg, H. Gentner, 1960, p. 333.

24 Según la doctrina canónica, en efecto, el sujeto activo de la costumbre es la Iglesia, entendida como comunidad de fieles o «pueblo de Dios»: P. PICOZZA, voz «Consuetudine. IV) Diritto canonico», Enciclopedia giuridica, vol. VIII, Roma, Istituto della Enciclopedia Italiana, 1988, p. 3.

25 Una huella de esta tesis se encuentra en el mismo KELSEN, allá donde la costumbre jurídica es descrita como «forma democrática de producción del Derecho», similar en este sentido a la autonomía privada: H. Kelsen, Teoria generale del diritto e dello Stato, cit., pp. 315-316. 
como la de pueblo y la de voluntad colectiva, es decir, recurre a ficciones que hacen opaca la referencia al término «costumbre jurídica». En segundo lugar, la opacidad de la referencia hace por lo menos aleatoria la verificación de la costumbre. Si se admite - como hipótesis, en aras de la discusión — que la voluntad popular o colectiva existe independientemente de las formas jurídicas y/o institucionales que permiten a tal voluntad adquirir un contenido, entonces no se ve cómo puede ser demostrado el hecho de que el pueblo, o la colectividad, quieran que la repetición de una cierta conducta sea conforme a Derecho.

\subsection{2. ¿La costumbre jurídica es una convención?}

Una explicación mucho más interesante del enforcement de una regla social, en la que consistiría la costumbre jurídica, es ofrecida por la noción de opinio necessitatis como expectativa de reciprocidad, desarrollada por B. CELANO en dos importantes ensayos dedicados a este tema ${ }^{26}$. Se trata de una propuesta explicativa que amerita atención particular, sea por sus contenidos sea por sus implicaciones. Según CELANO el elemento subjetivo de la costumbre

«consiste en la disposición, por parte de quienes participan [en la acción], a comportarse de una cierta manera en relación a los otros participantes, con la condición de que, sin embargo, los demás hagan lo mismo o de que, en general, correspondan, en algún modo, a la prestación obtenida. En este sentido, subsiste una costumbre cuando varios individuos se conforman a una regularidad de comportamiento porque se espera que también los demás se conformen y por la misma razón» ${ }^{27}$.

La hipótesis que guía esta caracterización de la opinio es entonces la siguiente: las costumbres jurídicas surgen en las situaciones en las cuales la elección de cada agente acerca de la conducta a realizar depende de la elección de los otros agentes involucrados en la misma acción y, por tanto, de las expectativas recíprocas de comportamiento. Siendo así las cosas, está justificado afirmar, según CELANO, que «las costumbres son convencionales» ${ }^{28}$ : la costumbre consistiría en el resultado de un acuerdo tácito entre los actores sociales, dirigido a coordinar su comportamiento en función de satisfacer el interés que cada quien tiene al actuar.

Fácilmente uno se da cuenta de que la intuición de CELANO es la de utilizar como esquema explicativo de la costumbre, el concepto de convención desarrollado por D. K. LEWIS ${ }^{29}$. En la perspectiva de LEWIS, en efecto, se da el caso de una convención allá en

26 B. Celano, «Consuetudini, convenzioni», en P. ComanducCI y R. GuAstini (eds.), Analisi e diritto 1995. Ricerche di giurisprudenza analitica, Torino, Giappichelli, 1995, pp. 35-87, trad. castellana por J. J. MoRESO, «Costumbres, convenciones», en B. CELANO, Dos estudios sobre la costumbre, cit., pp. 15-77.

${ }_{27}$ B. Celano, Costumbres, convenciones, cit., p. 35. Esta solución permitiría, según Celano, «saldar el análisis teórico-jurídico de la costumbre con el análisis de la formación espontánea de reglas sociales, elaboradas gracias a la adquisición de la teoría de la interacción estratégica» (ibid., p. 36). El criterio de la expectativa de reciprocidad ha sido introducido en el debate italiano sobre la costumbre jurídica por LAZZARO, que retoma el discurso de la antropología cultural y de la sociología: G. LAZZARO, «Reciprocità e consuetudine», en U. SCARPELLI (ed.), La teoria generale del diritto. Tendenze e problemi attuali. Studi dedicati a Norberto Bobbio, Milano, Comunità, 1983, pp. 231-247. Un modelo análogo ha sido esbozado por G. GAVAzZI, Elementi di teoria del diritto, Torino, Giappichelli, 1994², p. 104.

28 B. Celano, Costumbres, convenciones, cit., p. 57.

29 D. K. LewIs, Convention. A Philosophical Study, Cambridge (Mass.), Harvard University Press, 1969. 
donde están en juego problemas de coordinación entre dos o más individuos. Piénsese en el problema de elegir entre optar por la derecha o por la izquierda al circular en la vía pública, o el problema de establecer quién llama nuevamente en el caso de que una conversación telefónica se interrumpa ${ }^{30}$. Los problemas de coordinación, como muestran las teorías de los juegos, tienen la particularidad de admitir más soluciones. Los intereses de los agentes para no incurrir en un accidente en la vía pública, por ejemplo, es satisfecho sea optando por la derecha sea optando por la izquierda. Lo importante es que cada individuo involucrado tenga el mismo comportamiento, espere que los otros hagan lo mismo, prefiera que esto suceda, y tengan aproximadamente las mismas razones para hacerlo; éstas son, en breve, las condiciones necesarias y suficientes para resolver un problema de coordinación, es decir, en la perspectiva de LEWIS, para que nazca una convención ${ }^{31}$. Ahora, ¿qué determina la elección de la «posición de equilibrio» entre las conductas sociales fijadas por tal convención? La originalidad de la investigación de LEWIS —que permite a CELANO ampliar los resultados al estudio de la costumbre jurídica- consiste en la tesis según la cual la posición de equilibrio sería fijada sobre la base de los comportamientos llevados a cabo precedentemente en situaciones similares (precedent) ${ }^{32}$.

Supongamos que Magdalena y Cristina sepan que un cierto problema de coordinación - «qquién llama nuevamente si la comunicación telefónica se interrumpe?— ha sido resuelto en el pasado del mismo modo innumerables veces, o sea, aquella que había llamado primero ha sido siempre la que ha vuelto a llamar en el caso de que la comunicación se interrumpiera. Supongamos que ambas, tanto Magdalena como Cristina, sepan que saben que ésta ha sido en el pasado la solución exitosamente adoptada; supongamos además que ambas sepan que saben que saben que ésta ha sido la solución adoptada, y así al infinito ${ }^{33}$. Bien, en este caso subsiste una convención entre Magdalena y Cristina, la cual guiará sus comportamientos en este tipo de situación a menos que no existan razones relevantes para actuar de forma diferente. Según LEWIS, entonces, una convención se articula en dos momentos especulares, por un lado consiste en una regularidad de comportamiento que se repite sobre la base de las expectativas recíprocas de comportamiento y del conocimiento compartido que tengamos de ellas; por otro lado, una convención consiste en una serie de expectativas recíprocas de comportamiento, acompañado por el conocimiento común de las mismas, generada

30 Ibid., pp. 5-8.

31 Según LEWIS, en efecto, «a regularity $\mathrm{R}$ in the behavior of members of a population $\mathrm{P}$ when they are agents in a recurrent situation $\mathrm{S}$ is a convention if and only if it is true that, and it is common knowledge in $\mathrm{P}$ that, in any instance of $\mathrm{S}$ among members of $\mathrm{P}$,

(1) everyone conforms to $\mathrm{R}$;

(2) everyone expects everyone else to conform to $\mathrm{R}$;

(3) everyone has approximately the same preferences regarding all possible combinations of actions;

(4) everyone prefers that everyone conform to $\mathrm{R}$, on condition that at least all but one conform to $\mathrm{R}$; to $\mathrm{R}$,

(5) everyone would prefer that everyone conform to R', on condition that at least all but one conform

where $\mathrm{R}$ ' is some possible regularity in the behavior of members of $\mathrm{P}$ in $\mathrm{S}$, such that no one in any instance of $\mathrm{S}$ among members of $\mathrm{P}$ could conform both to R' and to R» (LEWIS, Convention, cit., p. 76).

32 El precedente es, en efecto, descrito por LEWIS como un tipo de «salience», o sea como la solución de un problema de coordinación, en ausencia de comunicación entre los agentes, que se diferencia de otras soluciones «by its uniqueness in some conspicuous respect» (ibid, p. 35). La unicidad bajo un perfil relevante de esta solución estaría en el hecho de que si una acción ha tenido lugar en el pasado, tendemos a repetirla en los casos similares «if we have no strong reason to do otherwise» (ibid., p. 37).

33 Es esta la situación descrita por LEWIS como «common knowledge» (ibid., pp. 52 y ss.). 
por la repetición de una conducta. Proyectando esta explicación sobre la costumbre jurídica, se puede entonces afirmar que

«subsiste una costumbre si: 1) la repetición general y constante de un cierto tipo de comportamiento produce la convicción de que la misma constituye un estándar vinculante de conducta; 2) la convención de que el tipo de comportamiento en cuestión constituye un estándar vinculante de conducta produce su repetición general y constante» ${ }^{34}$.

Se trata de una tesis muy interesante. En primer lugar explica cómo cierta regularidad de conducta se refuerza en el interior de un grupo social con el paso del tiempo, dando lugar así a costumbres, sin que con esto se recurra a una hipótesis contraintuitiva o a entidades ontológicamente dudosas. En segundo lugar, la tesis de CELANOLEWIS supera las paradojas de la derrotabilidad temporal y material. Esto se debe al hecho de que el requisito de la expectativa de reciprocidad presupone una «relación interna» entre el aspecto subjetivo y el aspecto objetivo de la costumbre, o sea entre la regla y los comportamientos que reflejan la conducta que ésta prescribe ${ }^{35}$. A partir de esta reconstrucción, efectivamente, ni el usus ni la opinio actúan como variables independientes en la explicación de la costumbre jurídica, como en cambio sucede en los modelos considerados precedentemente. El elemento material y el elemento psicológico se determinan recíprocamente a lo largo del continuum de la repetición ${ }^{36}$.

Aunque los escritos de CELANo hayan marcado, sin duda, un paso adelante en la comprensión de la costumbre jurídica, abriendo nuevos escenarios de investigación sobre este tema, la tesis de la expectativa de reciprocidad es insuficiente bajo múltiples perfiles. A continuación enumeramos los principales:

a) Según Celano, se da el caso de una costumbre jurídica si y sólo si, subsisten problemas de coordinación social, es decir, si el interés que uno tiene para actuar puede ser satisfecho en muchos modos diferentes pero su satisfacción depende de todos modos de las conductas de los otros. Ahora, admitiendo que ésta sea una condición necesaria para el nacimiento de una convención, se trata de una condición demasiado restrictiva para el nacimiento de una costumbre. Consideremos, una vez más, el caso del proveedor de servicios de Internet. La elección de los proveedores para garantizar la privacidad del registro de las conexiones del cliente no responde a exigencias de coordinación entre quien desarrolla esta profesión ${ }^{37}$. A diferencia de la circulación en la vía pública o del éxito de una comunicación telefónica, la actividad en cuestión depende sólo indirectamente de las conductas de quien realiza la misma actividad. La opinio necessitatis aquí parece surgir, más bien, de consideraciones diversas, que reenvían a la creencia de la obligatoriedad de reglas deontológicas, principios jurídi-

34 B. Celano, Costumbres, convenciones, cit., p. 44.

35 Retomo la noción de internal relation de G. BACKER y P. HACKeR, Wittgenstein. Rules, Grammar and Necessity, Oxford, Blackwell, 1994, pp. 98 y ss., quienes la utilizan para explicar la relación entre una regla y la práctica de seguir tal regla.

36 Se podría aquí objetar, sin embargo, que la tesis apenas formulada, aunque elegante desde el punto de vista filosófico, tiene dudosa capacidad explicativa. Ésta, en efecto, hace oscura la relación entre opinio y usus, resolviéndose de hecho en una variante de la teoría del error, o en una forma de naturalismo normativo.

37 Según A. MARMOR, efectivamente, la propuesta de LEWIS no es capaz de dar cuenta de algunos tipos importantes de convención, como por ejemplo las constitutive conventions, que no se dejan explicar en términos de soluciones prácticas a problemas de coordinación. Cfr. A. MARMOR, Positive Law and Objective Values, Oxford, Oxford University Press, 2001, pp. 1-24. 
cos, preceptos morales. El concepto de convención desarrollado por LEWIS no parece poder dar cuenta de este tipo de situaciones.

b) Tanto el análisis de LEWIS, como el análisis de CELANO, además, no dan cuenta del carácter normativo de costumbres y convenciones. Las investigaciones de estos autores no apelan a términos deónticos como «obligación», «prohibición», «permiso», etcétera, para explicar en qué consiste su objeto de estudio, ni parecen reconocer a aquél una función normativa ${ }^{38}$. Sin embargo, una costumbre jurídica no describe cómo los individuos se comportan sino cómo los individuos se deben comportar, las costumbres tienen un contenido prescriptivo, que hace de éstas un instrumento de regulación social. Una explicación satisfactoria de la costumbre jurídica que permita precisar las condiciones necesarias y suficientes para que exista tiene por tanto que dar cuenta de este aspecto fundamental. A esto se podría, en verdad, replicar que la costumbre es importante para el Derecho exclusivamente en cuanto becho considerado idóneo para producir normas jurídicas por medio de una norma sobre la producción de normas. Tal observación - aunque correcta según el perfil de la validez formal de las normas de formación consuetudinaria- contradice, sin embargo, la intuición que es base del requisito de la opinio necessitatis. Se trata de la idea de que una norma consuetudinaria consiste en el enforcement de una regla social, capaz de garantizar el reconocimiento, por parte del ordenamiento, del contenido normartivo de dicha regla a la luz de su obligatoriedad. La hipótesis explicativa de CELANO no preserva tal intuición, y parece más bien negar la subsistencia de una relación de tipo conceptual entre costumbre y normatividad ${ }^{39}$.

c) La falta de consideración de la función normativa de costumbres y convenciones hace que este análisis sea insuficiente bajo al menos dos perfiles. En primer lugar, la noción de convención no captura las condiciones suficientes para que un cierto tipo de conducta resulte predominante en un grupo social. El análisis de LEWIS explica en modo elegante cómo un tipo de conducta se consolida en el interior de un grupo, pero considera del todo causal, sorprendentemente, que tal conducta se imponga sobre las conductas alternativas que de todos modos garantizarían la coordinación social. Del mismo modo, la noción de costumbre propuesta por CELANO no está en posibilidad de especificar cuáles son las condiciones suficientes para que una regla social sea reconocida como una norma jurídica de formación consuetudinaria. ¿Cualquier regularidad calificada de conducta, acompañada por una expectativa de reciprocidad, produce una norma jurídica que prescribe tal conducta? Como ya hemos afirmado, la respuesta es obviamente negativa. Subsisten condiciones ulteriores para que esto suceda, y la noción de costumbre como convención no da cuenta de ellas.

d) La noción de costumbre como convención se contrapone a la opinión —aún hoy dominante en la doctrina, y no menos interesante bajo el perfil teórico- según la cual la costumbre es una consecuencia no intencional de la repetición de comportamientos intencionales. El Derecho consuetudinario surgiría en modo espontáneo,

38 «... "convention" itself, on my analysis, is not a normative term» (LEWIS, Convention, cit., p. 97). CELANO observa, por su parte, que «el "deber" que figura en la actitud de los participantes en la práctica consuetudinaria no es (...) un deber normativo, sino que es un deber técnico, o mejor la expresión de un nexo de racionalidad u oportunidad estratégica» (CELANO, Costumbres, convenciones, cit., p. 52).

39 En relación con el mismo problema en LEWIS, cfr. M. GILBERT, On Social Facts, New York, Routledge, 1989; A. MARMOR, «On Convention», Synthese, núm. 107, 1996, pp. 349-371. 
involuntario, anónimo, inconsciente ${ }^{40}$, sobre la base de comportamientos orientados a conseguir un resultado diverso del nacimiento de una norma jurídica. Objeto de la opinio, en otros términos, no sería la obligatoriedad de una conducta en tanto conforme a Derecho. La opinio necessitatis consistiría más bien en la creencia de que tal conducta constituya una condición necesaria para alcanzar algún fin que no ha de coincidir necesariamente con el fin perseguido por quien ha tenido el mismo comportamiento en el pasado o lo tendrá en el futuro, ni tampoco con el fin de cumplir lo prescrito por una norma jurídica ${ }^{41}$. La noción de convención utilizada por CELANO presupone, en cambio, que la regla consuetudinaria constituye el resultado de un acuerdo implícito entre los participantes en la acción ${ }^{42}$; es decir, se daría el caso de costumbres solamente a partir de elecciones conscientes y deliberadas, o sea en presencia de una intencionalidad colectiva orientada a la formación de una regla de conducta. Esta tesis, sin embargo, excluye del campo de atención de la ciencia jurídica y doctrina la costumbre involuntaria o no intencional ${ }^{43}$.

e) De lo recién señalado se sigue que concebir la costumbre como una convención vacía de contenido otras útiles distinciones elaboradas por la doctrina, como aquélla entre costumbres constitucionales y convenciones constitucionales ${ }^{44} \mathrm{o}$, haciendo referencia a los sistemas de common law, entre general custom y conventional custom ${ }^{45}$. Se trata de distinciones efectivamente controvertidas que permiten, sin embargo, precisar las características peculiares de algunas formas de regulación de los comportamientos sociales e institucionales que parecen sustraerse a la alternativa entre mera regularidad de comportamiento y convención.

\subsection{La costumbre jurídica como Derecho de producción judicial}

Al llegar a este punto de nuestra investigación, resulta evidente que los requisitos del usus y de la opinio son vagos y ambiguos; además, cualquier intento por determinar el contenido desencadena paradojas de no fácil solución o resulta de igual modo insatisfactorio. Se desprende que la verificación de la costumbre parece abrir amplios espacios de discrecionalidad en el operador jurídico: verificar si una conducta integra o no el caso de producción normativa «costumbre» parece configurarse, en realidad, como el acto constitutivo de la norma misma. Siendo así, está justificado afirmar que

40 S. Romano, Frammenti di un dizionario giuridico, Milano, Giuffrè, 1947, p. 45. Se trata de la conocida tesis con base en la cual el término «costumbre» se refiere al «mero hecho, general y anónimo (naturalistamente entendido, por así decir), que así haya sido y así vaya a ser» (CRISAFUlli, Lezioni di diritto costituzionale, cit., p. 180). Cfr. también Rossano, La consuetudine nel diritto costituzionale, cit., pp. 76-77.

${ }_{41}$ Cfr. M. BARBERIs, L'evoluzione del diritto, Torino, Giappichelli, 1997, pp. 248 y ss.

42 B. Celano, Costumbres, convenciones, cit., pp. 53-54 y 56-57.

43 Subrayan en cambio la relevancia de este tipo de costumbre, F. VIOLA y G. ZACCARIA, Le ragioni del diritto, Bologna, Il Mulino, 2003, p. 210.

44 Sobre las convenciones constitucionales, y sobre sus relaciones controvertidas con las costumbres constitucionales, por un lado, y la interpretación constitucional por el otro, reenvío al exhaustivo cuadro problemático ofrecido por G. U. RescignO, «Ripensando le convenzioni costituzionali», Politica del diritto, núm. 4, 1997, pp. 499-526. Sobre el punto, vid. también GUASTINI, Teoria e dogmatica delle fonti, cit., pp. 655-660; R. BIFULCO, «Riflessioni della cultura giuspubblicistica sulle convenzioni costituzionali», Diritto e società, núm. 1, 1992, pp. 31-55; G. ZAGREBELSKY, voz «Convenzioni costituzionali», Enciclopedia giuridica, vol. IX, Roma, Istituto della Enciclopedia Italiana, 1988; G. U. Rescigno, Convenzioni costituzionali, Padova, CEDAM, 1972 , en particular pp. 110 y ss.

45 Cfr. SHINER, Legal Institutions and the Sources of Law, cit., pp. 67-69. 
la costumbre jurídica consiste en aquello que el juez trata como tal en la solución de una controversia.

Es esta la tesis defendida, por ejemplo, por J. Austin y A. Ross, quienes conciben el Derecho consuetudinario como un Derecho de producción judicial. Como observa AUSTIN, en particular, esta tesis tiene la ventaja de evitar hacer referencia a cualquier «mysterious and imposing attribute» para reconocer intrínseca fuerza jurídica a la costumbre ${ }^{46}$. Porque «the sovereign, or supreme legislator, is the author of all the law» ${ }^{47}$, las costumbres son simples preceptos de moral positiva, que pueden ser convertidos en Derecho sólo por la autoridad delegada del juez ${ }^{48}$. Según Ross, en cambio, esta tesis desenmascara el contenido ideológico de la posición de quien reconoce aún gran importancia al Derecho consuetudinario, conservándole una posición autónoma en el sistema de las fuentes ${ }^{49}$. La noción de costumbre jurídica ciertamente es muy útil para comprender la evolución de las sociedades arcaicas y del Derecho primitivo. En los sistemas jurídicos contemporáneos, caracterizados por el primado indiscutible del Derecho legislativo, el término «costumbre» no denotaría, sin embargo, algún hecho normativo independiente - excepción hecha de las costumbres internacionales y, en ciertos aspectos, aquellas comerciales y de la navegación-. El uso del término «costumbre», en resumidas cuentas, serviría para «disimular la libertad del juez y su actividad creadora de Derecho» ${ }^{50}$.

La teoría de la costumbre como convención, por otra parte, llega a conclusiones del todo similares. Ya que las convenciones implícitas, acompañadas por la repetición del comportamiento objeto del acuerdo, no permiten, en cuanto tales, seleccionar los acuerdos que merecen el enforcement jurídico, se sigue que esta elección se deja a la apreciación discrecional del juez.

La hipótesis explicativa que estamos discutiendo, por tanto, parte de una acepción alternativa de la opinio, que se diferencia de las precedentes no por los contenidos sino por el sujeto al cual la opinio es imputada. La costumbre jurídica puede ser entendida como Derecho de producción judicial (o sea, el Derecho de producción judicial consiste en una forma de Derecho consuetudinario ${ }^{51}$ ) sólo si la opinio es imputada al juez y no en cambio a los destinatarios de la norma ${ }^{52}$.

${ }^{46} \mathrm{~J}$. Austin, Lectures on Jurisprudence or the Philosopby of Positive Law, R. CAMPBELL (ed.), London, J. Murray, $1885^{5}$, vol. I, p. 102. Aquí la objeción está dirigida a BLACKSTONE, quien había sostenido en 1765 que las costumbres «receive their binding power, and the force of law, by long and immemorial usage, and by their universal reception throughout the kingdom» (W. BLACKSTONE, Commentaries on the Laws of England, Philadelphia, Childs \& Peterson, 1860, vol. I, p. 63).

47 Austin, Lectures on Jurisprudence, cit., vol. II, p. 510.

48 Ibid., vol. I, p. 102.

49 Cfr. A. Ross, Diritto e giustizia, G. Gavazzi (ed.), Torino, Einaudi, 1990, pp. 88-89.

50 Ibid., p. 92. Sobre este punto, vid. también A. Ross, Theorie der Rechtsquellen. Ein Beitrag zur Theorie des positiven Rechts auf Grundlage dogmenhistorischer Untersuchungen, Leipzig-Wien, Deuticke, 1929, pp. 424 y ss.

51 Es este el caso de la doctrina alemana, en el ámbito de la cual la tesis aquí considerada es utilizada preferentemente no por las teorías de la costumbre sino por la teoría del Richterrecht, o sea del Derecho de producción judicial. Algunas fuentes típicas de Richterrecht, como por ejemplo el vínculo del precedente, encontraría legitimación en cuanto derecho de formación consuetudinaria. Sobre este debate, vid. F. MüLlER, «Richterrecht». Elemente einer Verfassungstheorie IV, Berlin, Dunker \& Humblot, 1986, pp. 111 y ss.; M. KRIELE, Theorie der Rechtsgewinnung —entwickelt am Problem der Verfassungs_ interpretation, Berlin, Duncker \& Humblot, 1976, pp. 245 y ss.; J. EsSER, «Richterrecht, Gerichtsgebrauch und Gewohnheitsrecht», en J. EsSER y H. THIEME (hrsg.), Festschrift für Fritz von Hippel zum 70. Geburtstag, Tübingen, J. C. B. Mohr, 1969, pp. 95-130.

52 Vid., sobre este punto, N. KorTGen, Probleme des Gewohnheitsrechts, Frankfurt a.M., P. Lang, 1993, pp. 182 ss. 
Esta posición teórica se aparta de las paradojas de la derrotabilidad temporal y material por razones semejantes y contrarias a aquellas consideradas en el párrafo precedente. En aquel caso tales paradojas eran superadas en virtud de la relación interna entre repetitio y opinio; en el caso que estamos considerando, éstas no se producen porque pierden cualquier relevancia conceptual tanto las conductas de los destinatarios de la norma como sus (presuntos) comportamientos intencionales.

También esta solución — como ya notaba N. BOBBIO ${ }^{53}$ - suscita, sin embargo, no pocas perplejidades y conduce a éxitos contraintiutivos.

En primer lugar, las costumbres de Derecho público y algunas formas de costumbre internacional (piénsese en los casos en los que los sujetos activos de la costumbre están dotados de potestad de imperio) son reconocidas como normas jurídicas vinculantes independientemente de la decisión de una corte. Por lo menos para estos tipos de costumbre, la tesis de la opinio como creencia de obligatoriedad por parte del juez no tiene ninguna capacidad explicativa.

En segundo lugar, es preciso preguntar: ¿el juez está obligado a aplicar la norma consuetudinaria así como lo prescribe una norma recavada de los textos normativos? Si una disposición normativa prescribe la aplicación de una norma consuetudinaria C, la falta de aplicación de $\mathrm{C}$ puede motivar la anulación de $\mathrm{S}^{54}$. Sin embargo, si la falta de aplicación de $\mathrm{C}$ es tratada como motivo de anulación de la sentencia $\mathrm{S}$, es contradictorio afirmar que $\mathrm{S}$ es condición de existencia $\mathrm{C}$.

En tercer lugar, la tesis de la opinio como creencia de obligatoriedad del juez no constituye, en realidad, una teoría de la costumbre jurídica, sino más bien, un argumento crítico que priva a esta teoría de su mismo objeto. Como oportunamente observa BOBBIO, «la teoría judicial o austiniana... más que explicar el Derecho consuetudinario, lo elimina» ${ }^{55}$. Dentro de los sistemas jurídicos contemporáneos, la costumbre jurídica no encontraría lugar alguno, ésta sería archivada como un tipo de objeto arqueológico, hoy vaciado de cualquier función regulativa.

Es ésta, sin embargo, una conclusión del todo contraintuitiva. Desde el momento que la costumbre jurídica, independientemente del comportamiento y de las creencias de los jueces, continúa desempeñando roles de cierta importancia en algunos sectores del Derecho, y es generalmente reconocida por los ordenamientos como fuente, nace la sospecha de que decretar su extinción sea la conclusión de un mal análisis conceptual ${ }^{56}$.

${ }^{3}$ Cfr. BobBIo, voz Consuetudine, cit., pp. 434-435.

54 Como recuerda oportunamente GUASTINI, «en virtud del art. 1, disp. prel. cod. civ., la violación de normas consuetudinarias, allá en donde estas son aplicables, constituye violación de derecho, y puede por tanto ser deducida como motivo de recurso de casación» (R. GUASTINI, Teoria e dogmatica delle fonti, Milano, Giuffrè, 1998, p. 649).

55 BobBIO, voz «Consuetudine», cit., p. 435. Se trata ésta de una tesis compartida por Celano quien, no casualmente, sostiene como conclusión de su investigación que «la costumbre no [puede] sensatamente, razonablemente, ser, hoy en día, fuente del Derecho» (B. CELANO, Costumbres y normas sobre la producción de normas, cit., p. 115).

56 Reevalúan en tal sentido el papel de la costumbre en la edad del «Derecho globalizado» VIOLA y ZACCARIA, Le ragioni del diritto, cit., pp. 209-210. 


\section{EL DOBLE ROSTRO DE LA COSTUMBRE JURÍDICA}

Como conclusión de esta investigación, es oportuno hacer una rápida alusión a los contextos de referencia de los usos del término «costumbre jurídica» utilizados en las tesis hasta aquí discutidas. Se trata de una alusión útil para especificar los presupuestos implícitos del discurso en torno a la costumbre jurídica y, por tanto, al origen de las paradojas que lo afectan.

Las asunciones que justifican los usos del término «costumbre jurídica» por parte de la ciencia jurídica y de la doctrina han sido tradicionalmente reconducidas a dos tesis alternativas la una de la otra:

a) El estudioso asume que la costumbre es fuente de producción normativa si, y sólo si, pertenece al ordenamiento una norma sobre la producción de normas (sea ésta explícita o implícita) que asume la costumbre como supuesto de hecho condicionante del nacimiento de una norma jurídica ${ }^{57}$. Desde esta perspectiva, como ocurre por ejemplo en el ordenamiento italiano, una previsión de ley en sentido genérico es condición de existencia de las normas de Derecho consuetudinario ${ }^{58}$.

Podremos denominar a este primer conjunto de asunciones: tesis de la normatividad extrinseca de la costumbre.

b) El estudioso asume que la costumbre jurídica puede producir normas jurídicas independientemente de cualquier previsión de la ley en sentido genérico. Es éste el caso de la llamada costumbre «extra ordinem» o costumbre como «fuente material» ${ }^{59}$, cuya idoneidad para producir normas jurídicas estaría condicionada no por otra norma jurídica sino por la «coherencia con la composición real de los poderes que se manifiestan y se estabilizan en un determinado contexto social» ${ }^{60}$. Debe tenerse en cuenta, para evitar equívocos, que en este caso la norma jurídica de formación consuetudinaria no se deduce de un hecho, de un estado de cosas o de un evento; es decir, no nos encontramos frente a un argumento que trae conclusiones normativas de premisas solamente descriptivas. Tras la existencia de una norma jurídica de formación consuetudinaria se encuentra o subyace, en realidad, una regla social constitutiva, puesta en la base de un cierto orden político-constitucional, la cual califica implícitamente este hecho, estado de cosas o evento como caso idóneo para producir normas jurídicas. La diferencia respecto a la tesis de la normatividad extrínseca de la costumbre, por tanto, reside en que la regla de reconocimiento de la costumbre sea una regla constitutiva de un orden social, no una norma jurídica sobre la producción

57 Cfr. KeLSEN, La dottrina pura del diritto, cit., pp. 18, 67 y 255.

58 Cfr. C. E. BAlossini, voz «Usi (Teoria degli)», Novissimo digesto italiano, vol. XX, Torino, UTET, vol. XX, 1975, pp. 200-209; V. CRISAFUlLI, «Variazioni sul tema delle fonti. Con particolare riguardo alla consuetudine», en VV.AA., Scritti in memoria di A. Giuffrè, Milano, Giuffrè, 1967, vol. III, pp. 253-277. Obsérvese, sin embargo, que una norma consuetudinaria es válida, además que existente, solamente si satisface condiciones ulteriores, por lo menos aquella de no ser contra legem.

59 Cfr. C. Esposito, voz «Consuetudine (diritto costituzionale)», Enciclopedia del diritto, vol. IX, Milano, Giuffrè, 1961, pp. 465-476; C. MorTATI, Istituzioni di diritto pubblico, Padova, CEDAM, 1962², p. 200.

60 G. ZaGREBELSKY, Manuale di diritto costituzionale. I: Il sistema delle fonti del diritto, Torino, UTET, 1988, p. 259. 
de normas; ésta no forma parte del ordenamiento, sino que lo hace posible desde una dimensión material.

Podremos denominar a este segundo conjunto de asunciones: tesis de la normatividad intrinseca de la costumbre.

Es posible proponer la hipótesis según la cual tanto el primer como el segundo conjunto de asunciones impiden desarrollar una teoría de la costumbre jurídica coherente, capaz de explicar las características del propio objeto. Basten aquí dos consideraciones críticas como justificación de esta hipótesis.

\subsection{Costumbre y regla de reconocimiento}

La tesis $a$ ) deja abierto el problema de la naturaleza y el fundamento de las normas sobre la producción de normas; es decir, de aquellas normas que califican a la costumbre como fuente del Derecho ${ }^{61}$. Se trata de un problema considerado hoy de escaso interés, que corre el riesgo de provocar formas de circularidad viciosa de ser el esquema explicativo de la costumbre que provee los instrumentos conceptuales para explicar en qué consiste una norma sobre la producción de normas ${ }^{62}$. Éste es el caso, por ejemplo, de quien explica la naturaleza y el contenido de este tipo de norma haciendo referencia a la noción hartiana de regla de reconocimiento.

Como es sabido, en la perspectiva de HART la regla de reconocimiento $\mathrm{R}$ prescribe que una norma $\mathrm{N}$ debe ser tratada como perteneciente al ordenamiento si y sólo si resultan satisfechos dos conjuntos de condiciones: 1) R es generalmente utilizada por los funcionarios como regla de conducta en la individuación de las normas que pertenecen al ordenamiento; 2) R está acompañada por la asunción del «punto de vista interno» por parte de los funcionarios, o sea de la creencia calificada que la conducta prescrita por $\mathrm{R}$ deba tenerse. Ahora bien, la aplicación de tales condiciones a las normas jurídicas de formación consuetudinaria se resuelve en el siguiente argumento: la costumbre jurídica consiste en un caso de producción normativa calificada como tal por la norma de reconocimiento; la norma de reconocimiento consiste en una costumbre jurídica; por tanto, la costumbre jurídica consiste en un caso de producción de normas calificada como tal por una costumbre jurídica ${ }^{63}$. Es ocioso observar que si ésta es la premisa implícita de la cual parte una teoría, entonces es irrazonable esperar de ella cualquier contribución para la explicación de su objeto.

${ }^{61}$ De la misma opinion, G. RoTOLO, «Sources of Law in the Civil Law», en SHINER, Legal Institutions and the Sources of Law, cit., p. 186.

62 Cfr. A. PeCzenik, On Law and Reason, Dordrecht, Springer, 1989, p. 331.

63 En el contexto del debate acerca de la regla de reconocimiento, el modo estándar de evitar el riesgo de circularidad consiste en considerar este argumento como una explicación genética más que como una explicación analítica; vid., entre muchos, J. Coleman, «The Practice of Principle», Defence of a Pragmatist Approach to Legal Theory, Oxford, Oxford University Press, 2001, pp. 100-102. Es posible replicar a esto sobre la base de lo que ha sido observado en relación con la teoría del error: prescindiendo de premisas ulteriores, una explicación genérica no está en posibilidad de explicar la naturaleza o la articulación de un concepto. Para una discusión más profunda del argumento de Coleman, vid., de todos modos, D. CANALE, «Vizi e virtù del pragmatismo concettuale», en P. COMANDUCCI y R. GuAstini (eds.), Analisi e diritto 2006, Torino, Giappiche1li, 2007, pp. 19-49. 


\subsection{Costumbre como fuente extra ordinem}

Con base en la tesis b), la costumbre jurídica desarrolla sólo en sentido derivado una función concurrente o complementaria respecto a otras fuentes. Ésta cumple por encima de todo una función constitutiva respecto a todas las fuentes del Derecho ${ }^{64}$. Tomar esta posición teórica tiene implicaciones singulares, que ameritan ser explicitadas.

En primer lugar, las características distintivas de la costumbre jurídica — la respuesta a la pregunta: ¿cómo debe ser una regularidad de comportamiento para producir normas jurídicas? - coinciden con las consecuencias derivadas de su aplicación. Si se concibe como norma constitutiva, en efecto, la costumbre jurídica instituye la situación que regula, haciendo plausible el juicio, frecuentemente enfatizado por la doctrina, según el cual es la efectividad de la norma consuetudinaria la que determina su existencia. De esto se sigue, también, que el contenido normativo de una costumbre es verificable solamente a posteriori, a través de la observación de aquellas regularidades de conducta que efectivamente han producido la norma consuetudinaria.

Pero si las cosas se dan en estos términos, asistimos a una radicalización de las paradojas de la derrotabilidad temporal y material consideradas al inicio. En primer lugar, cualquier conducta social se presta a representar la observancia de una norma consuetudinaria: es suficiente que tal conducta sea generalmente reconocida en un contexto social como dotada de autoridad. En segundo lugar, el contenido normativo de la costumbre jurídica funcionaría, por así decir, al contrario. Adoptando el léxico de SEARLE ${ }^{65}$, en lugar de tener una dirección de adaptación de mundo-al lenguaje, así como sucede para cualquier prescripción, la costumbre jurídica parecería caracterizada por una dirección de ajuste lenguaje-a-mundo, así como sucede para las descripciones. Es decir, el contenido prescriptivo de la costumbre se manifestaría, ex post factum, a través de la descripción de las conductas que reflejan la observancia de la norma consuetudinaria. En tercer lugar, todas las fuentes del Derecho, también las legislativas, podrían ser descritas nuevamente como una forma de Derecho consuetudinario, porque la validez de cualquier norma tendría fundamento consuetudinario.

Bien entendido, estas paradojas se prestan a ser fácilmente resueltas en el mismo momento de su formulación en un nivel u orden diferente del discurso. Por ejemplo, tratando la noción de costumbre jurídica como categoría antropológica, útil para explicar el funcionamiento de base de los comportamientos sociales, o bien considerándola como una categoría metafísica, capaz de representar el concepto mismo de normatividad $^{66}$.

${ }^{64}$ A la costumbre jurídica correspondería a veces «el rol de una norma de clausura, la cual, operando como fuente extra ordinem, produce las reglas fundamentales de un determinado ordenamiento jurídico», transformándose de esta manera «en una posible clave de interpretación de las transformaciones extralegales de los ordenamientos» [A. PIZzORUSSO, voz «Consuetudine (profili generali)», Enciclopedia giuridica, vol. VIII, Roma, Istituto della Enciclopedia Italiana, 1988, p. 1].

${ }^{65}$ Cfr. J. SeArle, «A Taxonomy of Illocutionary Acts», en K. Gunderson (ed.), Language, Mind, and Knowledge, Minneapolis, University of Minnesota Press, 1975, pp. 344-369.

66 Esta dirección de la investigación ha sido seguida, por ejemplo, por L. AsSIER-ADRIEU, «Il tempo e il diritto dell'identità collettiva. Il destino antropologico del concetto di consuetudine», Sociologia del diritto, 
Prescindiendo del interés filosófico suscitado por estos modos alternativos de aproximarse a nuestro tema, es cierto que aquellos hacen aún más vago y ambiguo el término «costumbre jurídica», haciéndolo de difícil provecho para el jurista. En otras palabras, la teoría de la costumbre jurídica corre el riesgo de transformarse en una doctrina filosófica que mantiene un contacto muy débil con los otros usos lingüísticos de los operadores del Derecho y con sus problemas cotidianos.

\section{CONCLUSIÓN}

He defendido en este ensayo que el discurso en torno a la costumbre jurídica formulado por la ciencia jurídica y por la doctrina no logra precisar a qué condiciones una regularidad calificada de comportamiento es fuente de una norma jurídica. Los intentos por precisar tales condiciones producen consecuencias sorprendentes: en ocasiones se generan paradojas; otras veces incurren en peticiones de principio; y otras veces llegan a conclusiones contraintuitivas, reduciendo las costumbres a fenómenos sociales diversos o incluso negando que las costumbres jurídicas pueden existir de verdad.

Si esto es así, entonces el pronóstico formulado al inicio de este ensayo cuenta con el apoyo de buenas razones: ciencia jurídica y doctrina son llamadas a redefinir el término «costumbre jurídica» con el fin de aclarar el papel de fuente del Derecho que a la costumbre se le sigue reconociendo. Al mismo tiempo, el análisis de las paradojas y de los problemas conceptuales subyacentes al estudio del Derecho consuetudinario muestra que este tema es aún susceptible de profundizaciones teóricas y reflexiones originales. Un trabajo éste que podría, tal vez, evidenciar cómo el concepto de costumbre, una vez liberado de las incrustaciones doctrinales que aún lo caracterizan, permite arrojar luz sobre la relación compleja que las normas jurídicas mantienen con otras formas de regulación social.

(Traducción de Edith Cuautle Rodríguez)

núm. 3, 1999, pp. 15-50; M. ATHANASIOS, «La forza della consuetudine», Rivista internazionale di filosofia del diritto, vol. 59, núm. 2, 1982, pp. 232-246; P. NolL, «Die Normativität als rechtsantropologisches Problem», en A. Bockelmann y U. K. KAUFMAnN (hrsg.), Festschrift für Karl Engisch zum 70. Geburtstag, Frankfurt a.M., Klostermann, 1969, pp. 125-142. 